\title{
Omega-3 fatty acids plus rosuvastatin improves endothelial function in South Asians with dyslipidemia
}

\author{
Catalin Mindrescu' ${ }^{1,2,3}$ \\ Rakesh P Gupta ${ }^{1,3}$ \\ Eileen $\vee$ Hermance' \\ Mary C DeVoe' \\ Vikas R Soma' \\ John T Coppola ${ }^{1,2}$ \\ Cezar S Staniloae ${ }^{1,2}$ \\ 'Comprehensive Cardiovascular \\ Center, Saint Vincent's Hospital \\ Manhattan, New York, NY, USA; \\ ${ }^{2}$ New York Medical College, Valhalla, \\ NY, USA; ${ }^{3}$ Rakesh P Gupta and Catalin \\ Mindrescu contributed equally \\ to this article.
}

Background: The present study was undertaken to investigate the effect of statins plus omega-3 polyunsaturated fatty acids (PUFAs) on endothelial function and lipid profile in South Asians with dyslipidemia and endothelial dysfunction, a population at high risk for premature coronary artery disease.

Methods: Thirty subjects were randomized to rosuvastatin $10 \mathrm{mg}$ and omega-3-PUFAs $4 \mathrm{~g}$ or rosuvastatin $10 \mathrm{mg}$. After 4 weeks, omega-3-PUFAs were removed from the first group and added to subjects in the second group. All subjects underwent baseline, 4-, and 8-week assessment of endothelial function and lipid profile.

Results: Compared to baseline, omega-3-PUFAs plus rosuvastatin improved endothelial-dependent vasodilation (EDV: $-1.42 \%$ to $11.36 \%, \mathrm{p}=0.001$ ), and endothelial-independent vasodilation (EIV: $3.4 \%$ to $17.37 \%, \mathrm{p}=0.002$ ). These effects were lost when omega-3-PUFAs were removed (EDV: $11.36 \%$ to $0.59 \%, p=0.003$ ). In the second group, rosuvastatin alone failed to improve both EDV and EIV compared to baseline. However, adding omega-3-PUFAs to rosuvastatin, significantly improved EDV ( $-0.66 \%$ to $14.73 \%, p=0.001)$ and $\operatorname{EIV~}(11.02 \%$ to $24.5 \%, p=0.001)$. Addition of omega-3-PUFAs further improved the lipid profile (triglycerides 139 to $91 \mathrm{mg} / \mathrm{dl}, \mathrm{p}=0.006$, low-density lipoprotein cholesterol 116 to $88 \mathrm{mg} / \mathrm{dl}, \mathrm{p}=0.014$ ).

Conclusions: Combined therapy with omega-3-PUFAs and rosuvastatin improves endothelial function in South Asian subjects with dyslipidemia and endothelial dysfunction.

Keywords: omega-3 fatty acids, endothelial function, South Asians, dyslipidemia, rosuvastatin

\section{Introduction}

Endothelium, as the major regulator of vascular homeostasis, exerts a number of vasoprotective effects, by maintaining the balance between vasodilation and vasoconstriction, inhibition and stimulation of smooth muscle cell proliferation and migration, and thrombogenesis and fibrinolysis (Vane et al 1990; Galley and Webster 2004). Many of these effects are largely mediated by nitric oxide (NO), the most potent endogenous vasodilator. A defect in the synthesis, release, or activity of nitric oxide leads to endothelial dysfunction, an early marker for atherosclerosis (Bonetti et al 2003). Endothelial function can be easily measured noninvasively with Doppler ultrasonography by assessing flow-mediated dilation (FMD) in the brachial artery after transient vascular occlusion (Faulx et al 2003; Corretti et al 2002). FMD serves as an index of NO bioavailability and vascular health.

The rapidly growing South Asian population in United States has one of the world's highest rates of endothelial dysfunction, metabolic syndrome and premature coronary artery disease (CAD) irrespective of region, religion, gender, and education. This may be a result of genetic predisposition to high levels of lipoprotein (a), 
small coronary arteries, low birth weight, in addition to environmental, nutritional and lifestyle factors (Bedi et al 2006; Gupta et al 2006).

Epidemiological studies and clinical trials indicate that the intake of very-long-chain n-3 polyunsaturated fatty acids (omega-3 PUFAs) such as eicosapentaenoic acid (EPA) and docosahexaenoic acid (DHA) reduces the risk of cardiovascular mortality (Bjerregaard and Dyerberg 1988; Newmans et al 1993; GISSI 1999). The cardioprotective effects of omega-3 PUFAs are widespread and are mediated by several mechanisms, which include decreasing triglyceride levels, increasing HDL levels, antiinflammatory properties, antithrombotic properties, antiarrhythmic properties and endothelial relaxation properties (Das 2000; Calder 2004).

Statins, potent inhibitors of cholesterol biosynthesis, are indicated in the primary and secondary prevention of CAD. Their overall benefits suggest effects beyond cholesterol lowering that involve improving endothelial function, enhancing the stability of atherosclerotic plaques, decreasing oxidative stress and inflammation and inhibiting the thrombogenic response (Liao and Laufs 2005).

The effect of a combined therapy of statins plus omega-3 PUFAs on endothelial function in a South Asian population, particularly at high risk for premature CAD, has not been investigated so far. We designed a randomized controlled cross-over study to test the hypothesis that omega-3 PUFAs intake plus rosuvastatin improves endothelial function in South Asians with dyslipidemia.

\section{Methods}

\section{Study population}

South Asian subjects (originating or having ancestry from India, Pakistan, or Bangladesh) now living in the New York metropolitan area were recruited for a protocol approved by our Institutional Review Board. Informed consent was obtained from each patient and the study protocol conforms to the ethical guidelines of the 1975 Declaration of Helsinki. To be eligible for the screening phase of the study, the subjects had to be 25 to 80 years old, and have a diagnosis of dyslipidemia, defined for the purpose of this study as a plasma low-density lipoprotein (LDL) cholesterol >100 mg/dl, and/or plasma triglyceride $>150 \mathrm{mg} / \mathrm{dl}$, with or without established CAD. All subjects deemed eligible after the screening phase underwent endothelial function assessment including endothelium-dependent vasodilation (EDV) and endothelium-independent vasodilation (EIV) with brachial artery ultrasound, as described below. Those who had an abnormal endothelial function, defined as an EDV less than
$7 \%$, were retained for the active phase of the study. Healthy arteries typically show an increase in diameter in the range of $5 \%$ to $20 \%$.

\section{Study design}

After the initial endothelial function assessment, patients were randomized in a crossover design between 2 drug regimens: rosuvastatin $10 \mathrm{mg}$ along with omega-3 fatty acid supplements (Fish oil capsules 4.53 g DHA/EPA, N3 Oceanic Inc., Palm, PA, USA) (Group 1) or rosuvastatin $10 \mathrm{mg}$ alone (Group 2) as shown in Figure 1. After 4 weeks, the omega-3 PUFAs supplements were removed from the treatment of Group 1 and added to the subjects in Group 2. Each patient served as his own control. The omega-3 fatty acid supplement capsules contained $430 \mathrm{mg}$ EPA, $325 \mathrm{mg}$ DHA, gelatin, glycerine, purified water, and natural lemon flavor as other ingredients. Subjects received 2 capsules three times per day. Patients taking statin medication were asked to stop that medication for four weeks before entering the study. All subjects maintained the same medication other than the study medication throughout the duration of the study. Compliance with the medication was assessed by pill count at each visit and at the end of the study.

\section{Assessment of endothelium-dependent and endothelium-independent vasodilation}

The endothelial function was evaluated according to the guidelines for the ultrasound assessment of flow-mediated vasodilatation of the brachial artery (Corretti et al 2002), as previously described from our laboratory (Barua et al 2002; Staniloae et al 2006). Although no gold standard for the measurement of endothelial function exist, the measurement of flow-mediated vasodilation in the brachial artery, assessed with Doppler ultrasound, has become the clinical technique of choice for the evaluation of endothelial function. The method was validated and widely used in numerous clinical studies (Faulx et al 2003; Sorensen et al 1995; Donald et al 2008). All measurements were performed early in the morning. Subjects were requested to abstain from any food or drink for at least 8 hours (overnight) and not use tobacco on the morning of the evaluation. All vasoactive medication was withheld on the morning of the study. On arrival subjects were allowed to rest in a supine position for 20 minutes. Baseline blood pressure and heart rate were recorded and blood was collected from the antecubital vein. This was followed by an in vivo assessment of endothelial function by using ultrasonography to measure the brachial artery diameter at rest, during reactive 


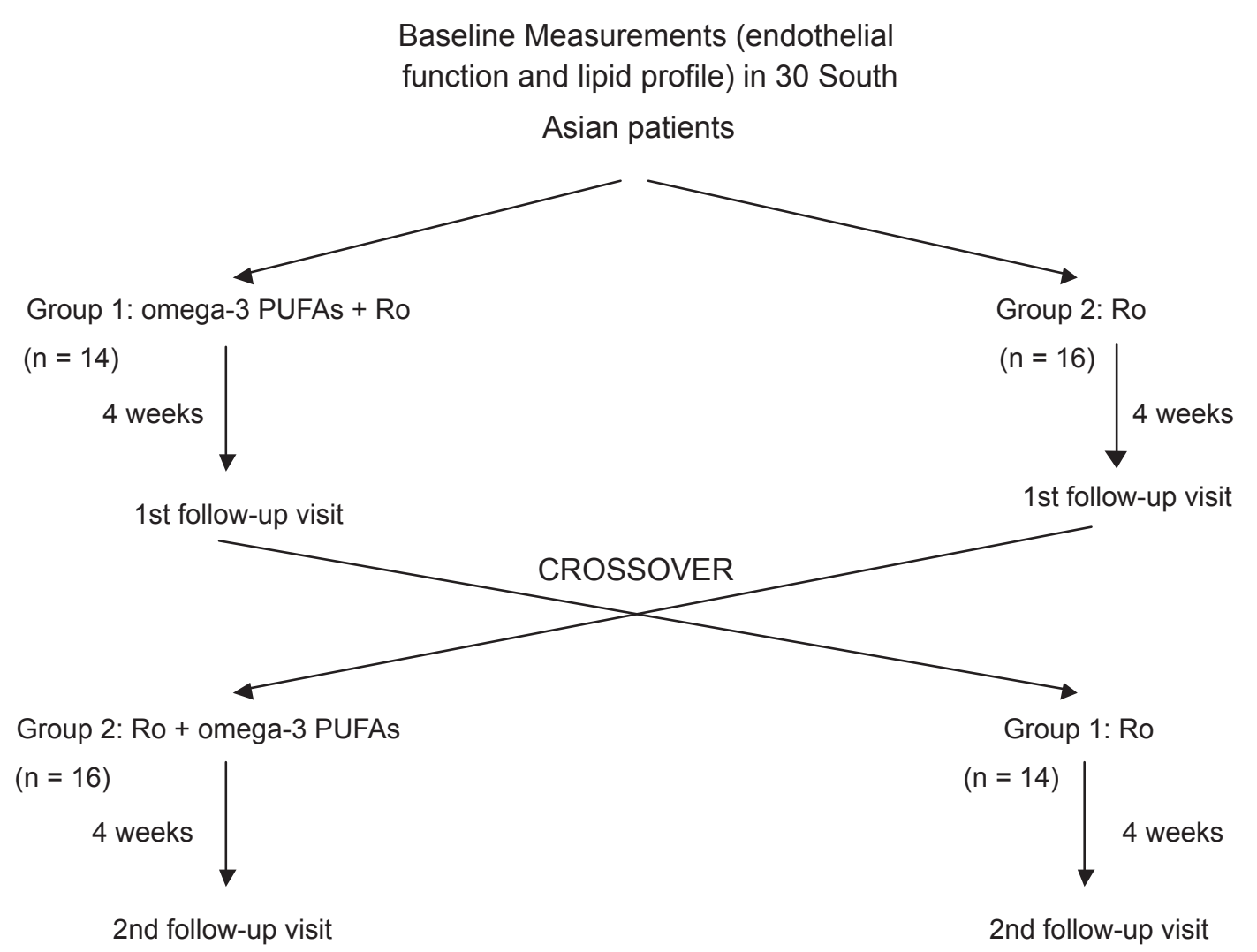

Figure I Study design:An open-label, randomized cross-over study. Group I represents patients starting with rosuvastatin plus omega-3 PUFAs, Group 2 represents patients starting with rosuvastatin only.

Abbreviations: Ro, rosuvastatin; Omega-3 PUFAs, Omega-3 polyunsaturated fatty acids.

hyperemia (leading to flow-mediated endothelium-dependent vasodilatation), and after administration of $0.4 \mathrm{mg}$ of sublingual nitroglycerin (an endothelium-independent vasodilator). After baseline imaging was performed by scanning the brachial artery in a longitudinal fashion, a pneumatic cuff was inflated around the right forearm to $50-60 \mathrm{mmHg}$ above the systolic pressure $(\approx 200 \mathrm{mmHg}$ ) for 5 minutes. The cuff was then released and the artery continuously imaged for 5 minutes. After an additional 5 minutes of recuperation, a new baseline imaging was obtained followed by the administration of $0.4 \mathrm{mg}$ nitrogycerin and continuously imaging for other 5 minutes. Flow-mediated endothelium dependent vasodilation (EDV) was calculated by dividing the maximum vessel diameter at 50 to 60 seconds after cuff deflation by the average baseline diameter. Endothelium-independent vasodilation (EIV) was measured as the maximum vessel diameter at 3 to 4 minutes after nitroglycerin administration divided by average baseline diameter. The arterial diameter was measured by using ultrasonic calipers for 4 cardiac cycles for each condition and then averaged. Flow-mediated vasodilation was expressed as the absolute change in diameter (in millimeters), and as the percent increase in diameter from baseline. All ultrasonographic scans were obtained by the same operator using the same equipment (Acuson L10 6- to 11-MHz transducer and Acuson Aspen System). All brachial artery scans were read by the same experienced ultrasonographer and validated by a second independent reader, both of whom were blinded to both the identity of the participants as well as the stage of the experiment. Across 100 observations performed in our laboratory by 2 observers, a diameter difference of $0.2 \mathrm{~mm}$ was estimated correctly in $72 \%$ of the cases. There was an interobserver variability of $0.1 \mathrm{~mm}$ in $28 \%$ of the cases. There was no difference larger than $0.2 \mathrm{~mm}$ between the 2 observers. The intraobserver variability was 0.82 .

\section{Laboratory measurements}

Fasting blood samples were obtained at baseline and at the end of each drug regimen (Week 4 and Week 8). Plasma analysis for the lipid profile, fasting glucose, C-reactive protein $(\mathrm{CRP})$, hemoglobin $\mathrm{A}_{1} \mathrm{C}\left(\mathrm{Hb} \mathrm{A}_{1} \mathrm{C}\right)$, erythrocyte sedimentation rate (ESR), and fibrinogen was done in the hospital's clinical laboratory. For the rest of the studies blood 
was collected in Vacutainer tubes for serum separation and centrifuged at $3000 \mathrm{rpm}$ for 15 minutes $\left(4^{\circ} \mathrm{C}\right)$. The serum was collected and stored at $-70{ }^{\circ} \mathrm{C}$ until use.

\section{Adiponectin ELISA}

Adiponectin concentrations in serum samples of patients participating in the study were measured using an ELISA kit (Linco Research, St. Charles, MO, USA) according to the manufacturer's instructions. Serum samples were diluted 500 times in the assay diluent buffer before analysis in order to fall within the dynamic range of the assay.

\section{Statistical analysis}

Analyses were performed with SPSS software (version 10.0; SPSS Inc., Chicago, IL, USA). Sample size calculations indicated that, to detect a mean difference in EDV of $2 \%$ on the study medication, using a croos-over design, 16 subjects would need to complete the study ( $\alpha$ statistic, 0.05 ; power $>0.8)$. Data are presented as mean $\pm \mathrm{SD}$. Wilcoxon signed-rank test was used to compare changes in outcome variables for endothelial function in response to treatment or with the wash out period. Paired or unpaired t test or chi square test were used to compare demographic baseline characteristics and laboratory values. Statistical significance was set for $\mathrm{p}<0.05$.

\section{Results}

Thirty of thirty-four screened subjects completed the study. Three subjects failed to fulfill eligibility criteria (endothelial function within normal range) and one was lost to follow-up. The study medication was well tolerated by all subjects and no bleeding or muscle symptoms were reported. Their mean age was 51 (range, 27 to 78 years). Their demographic and metabolic characteristics are summarized in Table1. The complete results of the assessment of the endothelial function are summarized in Table 3. For subjects in Group $1(n=14)$, compared to baseline, omega-3 PUFAs plus rosuvastatin improved endothelial-dependent vasodilation (EDV: $-1.42 \%$ to $11.36 \%, p=0.001$ ) (Figure 2 ), and endothelial-independent vasodilation (EIV: $3.4 \%$ to $17.37 \%$, $\mathrm{p}=0.002$ ) (Figure 3). When omega-3 PUFAs were removed, these effects were lost (EDV: $11.36 \%$ to $0.59 \%, p=0.003$ ) (Figure 2). In Group $2(\mathrm{n}=16)$, rosuvastatin alone failed to improve both EDV $(-2.49 \%$ to $-0.66 \%, \mathrm{p}=0.48)$ and EIV $(10.31 \%$ to $11.02 \%, \mathrm{p}=0.6)$ compared to baseline. However, when omega-3 PUFAs were added to rosuvastatin, there was a significant improvement in EDV $(-0.66 \%$ to $14.73 \%, \mathrm{p}=0.001)$ (Figure 2 ) and EIV (11.02\% to $24.5 \%$, $\mathrm{p}=0.001)$ (Figure 3). Addition of omega-3 PUFAs further improved the lipid profile in both groups (Figure 4). In group 1 total cholesterol decreased from 191 to $153 \mathrm{mg} / \mathrm{dl}$ $(\mathrm{p}=0.001)$, triglycerides from 139 to $91 \mathrm{mg} / \mathrm{dl}(\mathrm{p}=0.006)$,

Table I Baseline characteristics of the study subjects

\begin{tabular}{|c|c|c|c|}
\hline Characteristics & Group I $(n=14)$ & Group $2(n=16)$ & $P$ value \\
\hline Age (years), mean (range) & $5 \mathrm{I}(27-78)$ & $52(28-73)$ & NS \\
\hline Gender: Male (n, \%) & $12(86)$ & II (69) & NS \\
\hline Female (n, \%) & $2(14)$ & $5(3 I)$ & NS \\
\hline BMI, mean $\left(\mathrm{kg} / \mathrm{m}^{2}\right)$ & $27 \pm 4.2$ & $27.2 \pm 4.7$ & NS \\
\hline Dyslipidemia (n, \%) & $14(100)$ & $16(100)$ & NS \\
\hline Metabolic syndrome (n, \%) & II (79) & $16(100)$ & NS \\
\hline $\operatorname{CAD}(\mathrm{n}, \%)$ & $9(64)$ & II (69) & NS \\
\hline Diabetes (n, \%) & $5(36)$ & $5(3 I)$ & NS \\
\hline $\operatorname{HTN}(\mathrm{n}, \%)$ & $9(64)$ & $12(75)$ & NS \\
\hline \multicolumn{4}{|l|}{ Medications } \\
\hline $\operatorname{ASA}(n, \%)$ & $9(64)$ & $13(8 \mid)$ & NS \\
\hline Clopidogrel (n, \%) & $7(50)$ & $8(50)$ & NS \\
\hline Beta blocker (n, \%) & $9(64)$ & $9(56)$ & NS \\
\hline ACE inhibitor or ARB $(n, \%)$ & $7(50)$ & $10(63)$ & NS \\
\hline Statin & $\begin{array}{l}\text { 4-week washout prior } \\
\text { to randomization }\end{array}$ & $\begin{array}{l}\text { 4-week washout prior to } \\
\text { randomization }\end{array}$ & \\
\hline
\end{tabular}

Notes: Group I represents patients starting with rosuvastatin plus omega-3 PUFAs, Group 2 represents patients starting with rosuvastatin only. Data are presented as $n$ or mean \pm SD. Comparison between groups was done by chi-square test or t-test for age and BMI.

Abbreviations: NS, nonsignificant; BMI, body mass index; CAD, coronary artery disease; HTN, hypertension; ASA, aspirin; ACE, angiotensin-converting enzyme; ARB, angiotensin receptor blocker; PUFAs, polyunsaturated fatty acids; SD, standard deviation. 

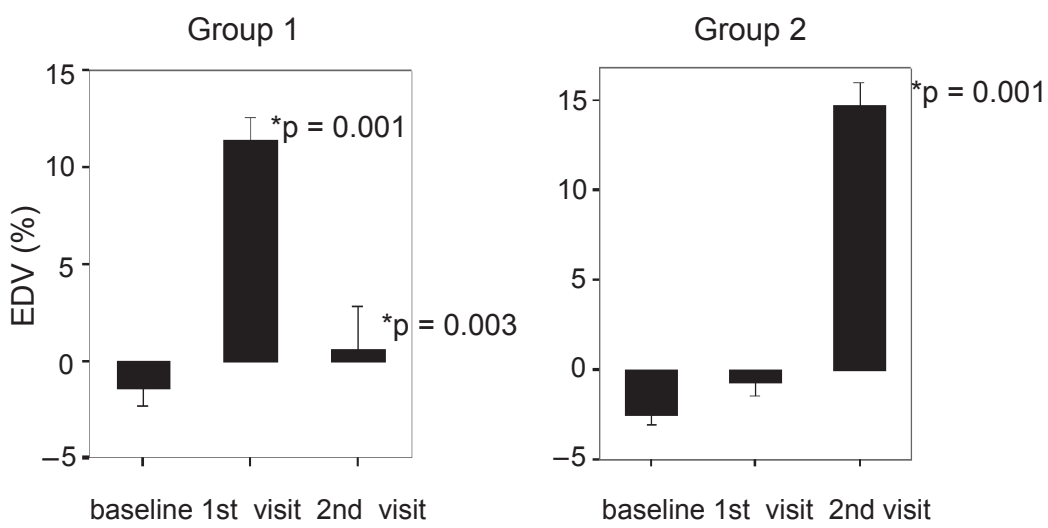

Figure 2 Average endothelial dependent vasodilation percentage (EDV\%) for groups I and 2 during each visit. Group I represents patients starting with rosuvastatin plus omega-3 PUFAs, Group 2 represents patients starting with rosuvastatin only. Data represent mean per group \pm SE.

Notes: *indicates statistical significance difference of average EDV\% between each visit by Wilcoxon test.

and LDL 116 to $88 \mathrm{mg} / \mathrm{dl}(\mathrm{p}=0.014)$. In group 2 total cholesterol decreased from 184 to $139 \mathrm{mg} / \mathrm{dl}(\mathrm{p}<0.001)$, triglycerides from 141 to $103 \mathrm{mg} / \mathrm{dl}(\mathrm{p}=0.013)$, and LDL from 109 to $76 \mathrm{mg} / \mathrm{dl}(\mathrm{p}=0.002)$. There were no significant differences in the CRP, ESR, and fibrinogen levels (Table 2). Nevertheless a tendency of decreasing in ESR was noted in group 2 without reaching statistical significance. Neither rosuvastatin nor the combination treatment affected the levels of adiponectin (Table 2).

\section{Discussion}

This study illustrates that the addition of omega-3 PUFAs to rosuvastatin in a high-risk population (South Asians) with dyslipidemia improves EDV and EIV as well as the lipid profile. Withdrawal of omega-3 PUFAs treatment is associated with a return of the endothelial function back to the baseline, in spite of continuing the treatment with rosuvastatin.
Several studies have shown that South Asians are an emerging risk group for $\mathrm{CAD}$ as compared with other ethnicities (Bedi et al 2006; Gupta et al 2006). Numerous factors, including a high prevalence of conventional risk factors (smoking, diabetes, hypertension, etc.), insulin resistance, metabolic syndrome, low birth weights, and genetic predisposition, have been shown to be responsible for this. The INTERHEART case-control study found that the population-attributable risk in the South Asian region is $58.7 \%$ for dyslipidemia, 37.4\% for smoking, $37.7 \%$ for abdominal obesity, $29.6 \%$ for hypertension, $11.8 \%$ for diabetes, and $15.9 \%$ for psychological risk factors (Yusuf et al 2004). Our study group was representative of this high-risk population: $100 \%$ of patients were dyslipidemic, $90 \%$ had metabolic syndrome (as defined by the ATP III criteria. (NCEP 2001), 70\% had established CAD, 70\% had hypertension, and $33 \%$ had a diagnosis of diabetes mellitus. Ninety percent of the patients screened for this study had severe endothelial dysfunction displaying a non-response or
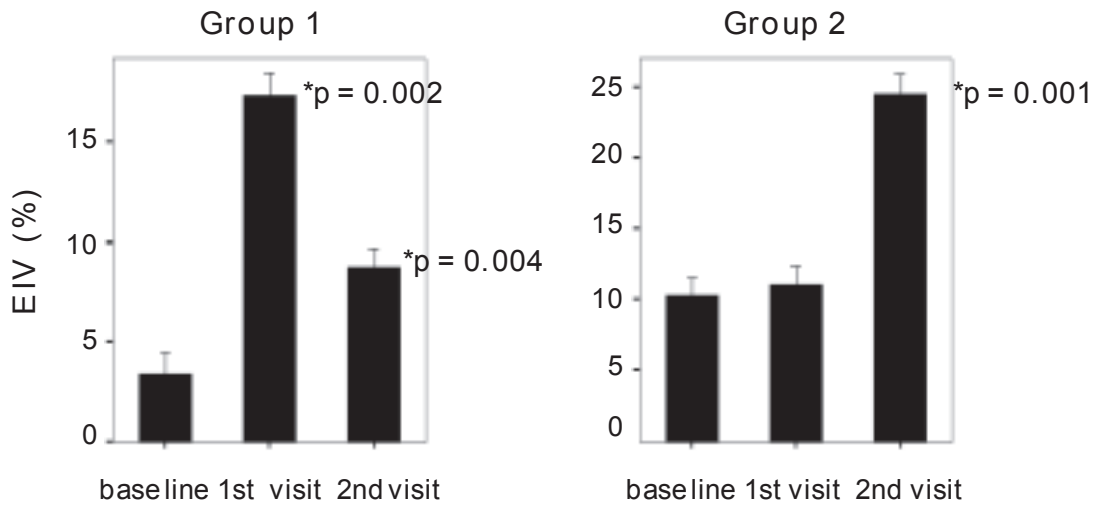

Figure 3 Average endothelial independent vasodilation percentage (EIV\%) for groups I and 2 during each visit. Group I represents patients starting with rosuvastatin plus omega-3 PUFAs. Group 2 represents patients starting with rosuvastatin only. Data represent mean per group $\pm \mathrm{SE}$.

Note: *indicates statistical significance difference of average EDV\% between each visit by Wilcoxon test. 

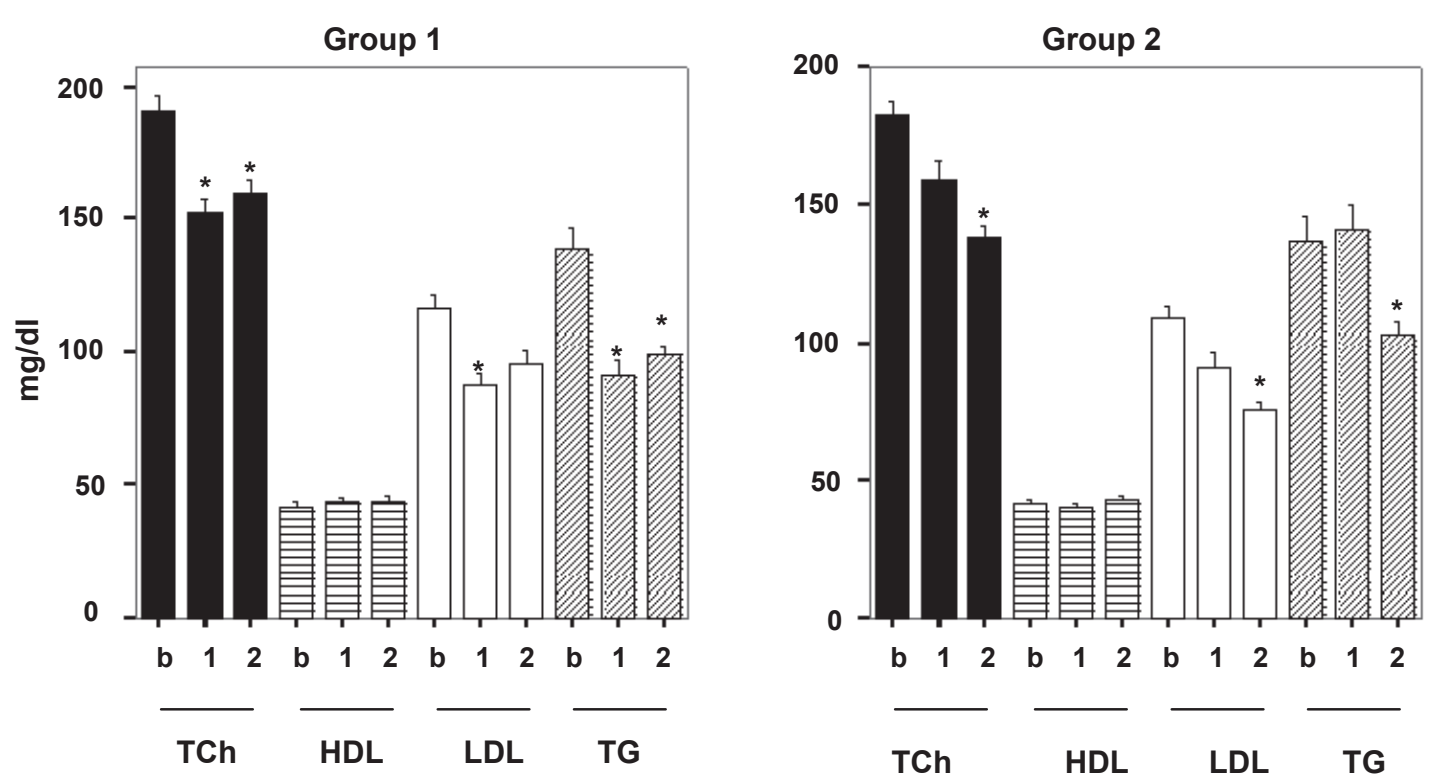

Figure 4 Lipid profile (including total cholesterol (TCh), high density lipoprotein (HDL), low density lipoprotein (LDL), and triglycerides (TG) for groups I and 2 at baseline (b), visit I (I), and visit 2 (2). Group I represents patients starting with rosuvastatin plus omega-3 PUFAs. Group 2 represents patients starting with rosuvastatin only. Data represent mean per group $\pm \mathrm{SE}$.

Note: *indicates statistical significance (by $t$ test) between each visit.

a vasoconstrictive response to shear stress. No studies to date have evaluated the effect of statins or omega-3 PUFAs on the endothelial function in this population.

Endothelial dysfunction is thought to be an early and important event in the development of many cardiovascular diseases including atherosclerosis, hypertension, and heart failure. The precise mechanism of endothelial dysfunction is not completely understood, but it is generally believed to be mediated by NO produced by the endothelial cells. Other mediators such as prostanoids are also involved. The potential for reversal of endothelial dysfunction with different therapies has generated considerable interest. Previous studies have yielded conflicting results about the effect of statins on endothelial function. Several studies report that statin therapy improves endothelial-dependent vasodilation in hypercholesterolemic patients (Leung et al 1993; Treasure et al 1995; Dupuis et al 1999). A

Table 2 Laboratory values

\begin{tabular}{|c|c|c|c|c|c|c|}
\hline & \multicolumn{3}{|l|}{ Group I } & \multicolumn{3}{|l|}{ Group 2} \\
\hline & Baseline & I st visit & 2nd visit & Baseline & I st visit & 2nd visit \\
\hline Total cholesterol (mg/dl) & $|9| \pm 44$ & $153 \pm 37 *$ & $159 \pm 35^{*}$ & $184 \pm 40$ & $159 \pm 54$ & $|39 \pm 3|^{*}$ \\
\hline $\mathrm{HDL}(\mathrm{mg} / \mathrm{dl})$ & $42 \pm 12$ & $43 \pm 13$ & $44 \pm 14$ & $41 \pm 15$ & $40 \pm 10$ & $43 \pm 15$ \\
\hline LDL (mg/dl) & $116 \pm 37$ & $88 \pm 29 *$ & $96 \pm 36$ & $109 \pm 31$ & $91 \pm 41$ & $76 \pm 24^{*}$ \\
\hline Triglycerides (mg/dl) & $139 \pm 57$ & $91 \pm 40^{*}$ & $99 \pm 27^{*}$ & $|37 \pm 7|$ & $|4| \pm 7 \mid$ & $103 \pm 36 *$ \\
\hline Glucose (mg/dl) & $124 \pm 54$ & $130 \pm 62$ & $112 \pm 30$ & $104 \pm 24$ & $108 \pm 39$ & $110 \pm 38$ \\
\hline Hemoglobin $A_{1} C(\%)$ & $7.0 \pm 2.2$ & $7.0 \pm 2.2$ & $6.6 \pm 1.8$ & $6.5 \pm 1.1$ & $6.3 \pm 1.0$ & $6.2 \pm 1.0$ \\
\hline Fibrinogen (mg/dl) & $357 \pm 72$ & $348 \pm 70$ & $338 \pm 68$ & $357 \pm 87$ & $352 \pm 70$ & $333 \pm 69$ \\
\hline C-reactive protein $(\mathrm{mg} / \mathrm{l})$ & $0.36 \pm 0.4 \mathrm{I}$ & $0.3 \pm 0.36$ & $0.34 \pm 0.46$ & $0.4 \pm 0.46$ & $0.25 \pm 0.23$ & $0.19 \pm 0.17$ \\
\hline $\operatorname{ESR}(\mathrm{mm} / \mathrm{hr})$ & $16 \pm 13$ & $14 \pm 12$ & $12 \pm 9$ & $18 \pm 13$ & $14 \pm 1 \mid$ & $9 \pm 9$ \\
\hline Adiponectin $(\mu \mathrm{g} / \mathrm{ml})$ & $8.63 \pm 3.56$ & $9.06 \pm 4.75$ & $8.98 \pm 4.16$ & $6.46 \pm 4.4$ & $6.22 \pm 3.98$ & $6.43 \pm 4.6$ \\
\hline BP Systolic (mmHg) & $119 \pm 10$ & $112 \pm 12$ & $122 \pm 16$ & $127 \pm 22$ & $13 \mid \pm 17$ & $132 \pm 16$ \\
\hline BP Diastolic (mmHg) & $71 \pm 7$ & $69 \pm 10$ & $77 \pm 9$ & $75 \pm 11$ & $80 \pm 10$ & $77 \pm 5$ \\
\hline
\end{tabular}

Notes: Group I represents patients starting with rosuvastatin plus omega-3 PUFAs. Group 2 represents patients starting with rosuvastatin only. Data are presented as mean \pm SD. Statistically significant differences are indicated with an asterisk.

Abbreviations: HDL, high-density lipoprotein; LDL, low-density lipoprotein; ESR, erythrocyte sedimentation rate; BP, blood pressure; PUFAs, polyunsaturated fatty acids; SD, standard deviation. 
number of mechanisms have been proposed to explain these effects. One important pathway appears to be the effects of statins on NO production via increased availability of endothelial nitric oxide synthase (eNOS). Thus, statins prevent the downregulation of eNOS mRNA by oxidized-LDL, hypoxia, and TNF- $\alpha$ (Hernandez-Perera et al 1998; Laufs et al 1998). Additionally HMG-CoA inhibition results in the activation of protein kinase Akt and the posttranslational phosphorylation of the eNOS protein (Dimmeler et al 1999; Fulton et al 1999). Nevertheless, other studies, particularly in subjects with type II diabetes, but also in patients with familial hypercholesterolemia, report no effect at all (Sheu et al 1999; Van de Ree et al 2001; van Etten et al 2002; ter Avest et al 2005). The reason for this inconsistency is unclear but could be related to the dependency of endothelial function on insulin resistance. There is also the possibility of a particular resistance to statins in South Asians and this finding deserves further investigation. Also, we cannot exclude a delayed vasodilative effect of rosuvastatin, but this is unlikely, as other studies have demonstrated a very early onset of the statin activity (Omori et al 2002). Similar findings have been reported by ter Avest and colleagues (2005) who showed no effect of rosuvastatin on endothelial function, in spite of a clear effect on the lipid profile and inflammatory markers.

Long-chain omega-3 PUFAs, found in fatty fish and fish oil have cardiovascular protective properties. They cause

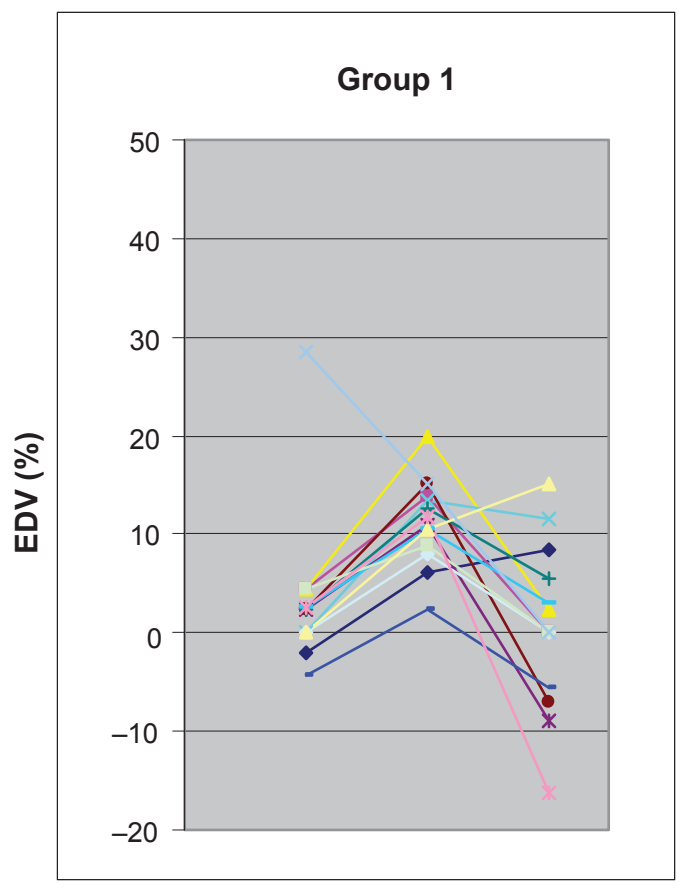

Baseline 1st visit 2nd visit endothelial relaxation and promote vascular compliance (Goode et al 1997; Tagawa et al 1999). Our study supports an early and consistent improvement in endothelium-dependent dilatation with treatment with the omega-3 PUFAs in South Asian patients with dyslipidemia treated with rosuvastatin (Figure 5). These findings extend prior observations that omega-3 PUFAs improve endothelium-dependent dilatation in hypercholesterolemic patients (Engler et al 2004). The effect may be due particularly to the DHA component of the fish oil capsules (Mori et al 2000). The mechanism is not fully understood, but omega-3 PUFAs may protect against vasospasm and thrombosis by enhancing NO release, and prostacyclin synthesis, and by suppressing thromboxane formation (McVeigh et al 1993).

Previously, it was proposed that omega-3 PUFAs mediate the actions of various statins and states of essential fatty acids deficiency are accompanied by an increased HMG-CoA reductase activity, making statins unable to lower plasma lipid levels (Das 2001). These observations could explain why we found the improvement of endothelial function in the presence of omega-3-PUFAs.

The present study showed, for the first time, that omega-3 PUFAs improved endothelial function in a group of subjects who appeared resistant to the vasoprotective effect of rosuvastatin. These findings support a therapeutic

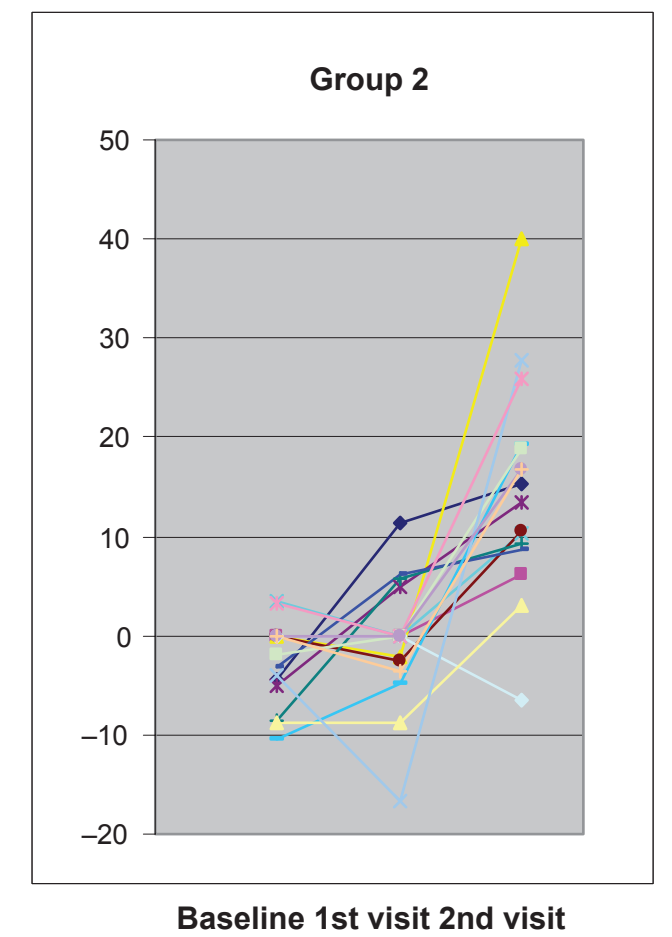

Figure 5 Plotted results of endothelium dependent vasodilation percentage (EDV\%) for each individual patient in group I and 2 between each visit. Group I represents patients starting with rosuvastatin plus omega-3 PUFAs, Group 2 represents patients starting with rosuvastatin only. 
Table 3 Assessment of endothelial function by brachial artery ultrasound

\begin{tabular}{|c|c|c|c|c|c|c|}
\hline & Group I & & & Group 2 & & \\
\hline Diameter (mm) & Baseline & Ist visit & 2nd visit & Baseline & Ist visit & 2nd visit \\
\hline Resting & $3.9 \pm 0.6$ & $3.7 \pm 0.7$ & $3.7 \pm 0.7$ & $3.7 \pm 0.7$ & $3.6 \pm 0.6$ & $3.1 \pm 0.6$ \\
\hline $\begin{array}{l}\text { Post-ischemic } \\
\text { maximum }\end{array}$ & $3.9 \pm 0.5$ & $4.1 \pm 0.7$ & $3.7 \pm 0.7$ & $3.6 \pm 0.8$ & $3.6 \pm 0.7$ & $3.6 \pm 0.7$ \\
\hline Absolute EDV & $-0.06 \pm 0.13$ & $0.4 I \pm 0.17^{*}$ & $0.007 \pm 0.31$ & $-0.09 \pm 0.15$ & $-0.006 \pm 0.21$ & $0.44 \pm 0.3 I^{*}$ \\
\hline$\%$ EDV & $-1.42 \pm 3.27$ & $11.36 \pm 4.33 *$ & $0.59 \pm 8.32$ & $-2.49 \pm 4.16$ & $-0.66 \pm 6.44$ & $14.73 \pm 10.77^{*}$ \\
\hline Post-nitroglycerine & $3.9 \pm 0.8$ & $4.2 \pm 0.8$ & $4.0 \pm 0.7$ & $3.9 \pm 0.6$ & $4.0 \pm 0.7$ & $4.0 \pm 0.8$ \\
\hline Absolute EIV & $0.11 \pm 0.23$ & $0.59 \pm 0.26^{*}$ & $0.3 \pm 0.27$ & $0.33 \pm 0.32$ & $0.38 \pm 0.36$ & $0.77 \pm 0.33^{*}$ \\
\hline \% EIV & $3.4 \pm 7.67$ & $17.37 \pm 9.27 *$ & $8.71 \pm 7.67$ & $10.31 \pm 9.62$ & $11.02 \pm 10.43$ & $24.5 \pm 11.94 *$ \\
\hline
\end{tabular}

Notes: Group I represents patients starting with rosuvastatin plus omega-3 PUFAs. Group 2 represents patients starting with rosuvastatin only. Data are presented as mean \pm SD. Statistically significant differences are indicated with an asterisk.

Abbreviations: EDV, endothelial-dependent vasodilation; EIV, endothelial-independent vasodilation; PUFAs, polyunsaturated fatty acids; SD, standard deviation.

strategy where omega-3 PUFAs should be added to a statin, particularly if the endothelial function fails to improve.

Flow-mediated dilatation of the brachial artery correlates well with coronary response to the endothelium-dependent vasodilator acetylcholine. Therefore, the use of omega-3 PUFAs supplements might possibly represent another therapeutic modality for patients with known, or at risk for coronary artery disease.

Our study confirms prior reports from animal models showing omega-3 fatty acid supplementation improves EIV (Engler et al 1994). The improvement in EIV was seen in both groups but only as an effect of omega-3 PUFAs (Figure 3). The removal of omega-3 PUFAs from the treatment returned the EIV towards baseline. Engler and colleagues (1994) reported that in aortic rings from spontaneously hypertensive rats and Wistar-Kyoto rats, EPA and DHA induced endothelium-dependent and-independent vasodilation, respectively. These may be attributable to direct and indirect effects of DHA on the arterial wall. The selective incorporation of DHA into endothelial membranes could increase membrane fluidity, calcium influx, and endogenous synthesis and NO release. Although we did not see a significant effect on the systolic or diastolic blood pressure, possibly because of the small sample size, EIV seems to be responsible for the improvement in blood pressure control with omega-3 PUFAs reported by others (Mori et al 2000).

This study is the first to show that the benefits of omega-3 PUFAs are rapidly lost with the withdrawal of treatment. Within 4 weeks of removal of omega-3 PUFA, the improvement in endothelial function was lost and the endothelial function, as measured by the EDV, returned to levels similar to the baseline. Moreover, this significant change in endothelial function was accompanied by worsening of EIV.
The use of statins is the standard of care for patients with dyslipidemia and at high risk for CAD. The study subjects exhibited an appropriate response to rosuvastatin (17\%-25\% reduction in LDL levels), which also confirms their compliance with the study medication. The addition of omega-3 fatty acid supplements to rosuvastatin led to further decreases in LDL values and significant decrease in the triglycerides levels. Nevertheless, none of the changes in the lipid profile correlated to the change in the EDV/EIV, suggesting that the effect of omega-3 PUFAs on endothelial vasodilation is mediated via other mechanisms. Although there are reports of an effect of omega-3 PUFAs on the level of adiponectin in rats (Flachs et al 2006) we found no significant alteration in the serum levels of adiponectin in our group of patients.

This study is limited particularly by the small sample size and by the relatively short period of treatment. Should these patients be treated longer, we could have possibly seen an effect of the rosuvastatin on the endothelial function. Nevertheless, it is encouraging to see an early and strong effect of omega-3 PUFA when added to the statin. Other limitation of our study is the lack of control group treated with omega3-PUFAs only. Although our findings strongly suggest that the improvement of endothelial function is due mainly to the omega-3-PUFAs, the results would have been more decisive having that control group.

In conclusion the addition of omega-3 PUFAs to rosuvastatin improves endothelial dependent and independent vasodilatation as well as further improves the lipid profile in South Asians with dyslipidemia. These vasodilative effects are lost with the removal of omega-3 PUFAs. Population studies and clinical trials provide compelling evidence that omega-3 PUFAs have significant cardioprotective effects and justify their use in the prevention of cardiovascular diseases. 
These benefits are achieved without the known side effects of other drug combinations. The present study also suggests that omega-3 PUFA's are not only effective triglyceride-lowering agents but also improve endothelial function in a group of subjects resistant to the vasoactive effect of rosuvastatin.

\section{Disclosure}

The authors report no conflicts of interest in this work.

\section{References}

Barua RS, Ambrose JA, Eales-Reynolds LJ, et al. 2002. Heavy and light cigarette smokers have similar dysfunction of endothelial vasoregulatory activity: an in vivo and in vitro correlation. $\mathrm{J} \mathrm{Am} \mathrm{Coll} \mathrm{Cardiol,}$ 39:1758-63.

Bedi US, Singh S, Syed A, et al. 2006. Coronary artery disease in South Asians: an emerging risk group. Cardiol Rev, 14:74-80.

Bjerregaard P and Dyerberg J. 1988. Fish oil and ischaemic heart disease in Greenland. Lancet, 2:514.

Bonetti PO, Lerman LO, Lerman A, et al. 2003. Endothelial dysfunction: a marker of atherosclerotic risk. Arterioscler Thromb Vasc Biol, 23:168-75.

Calder PC. 2004. n-3 Fatty acids and cardiovascular disease: evidence explained and mechanisms explored. Clin Sci (Lond), 107:1-11.

Corretti MC, Anderson TJ, Benjamin EJ, et al. 2002. International Brachial Artery Reactivity Task Force. Guidelines for the ultrasound assessment of endothelial-dependent flow-mediated vasodilation of the brachial artery: a report of the International Brachial Artery Reactivity Task Force. J Am Coll Cardiol, 39:257-65.

Das UN. 2000. Beneficial effect(s) of n-3 fatty acids in cardiovascular diseases: but, why and how? Prostaglandins Leukot Essent Fatty Acids, 63:351-62.

Das UN. 2001. Essential fatty acids as possible mediators of the actions of statins. Prostaglandins Leukot Essent Fatty Acids, 65:37-40.

Dimmeler S, Fleming I, Fisslthaler B, et al. 1999. Activation of nitric oxide synthase in endothelial cells by Akt-dependent phosphorylation. Nature, 399:601-5.

Donald AE, Halcox JP, Charakida M, et al. 2008. Methodological approaches to optimize reproducibility and power in clinical studies of flow-mediated dilation. J Am Coll Cardiol, 51:1959-64.

Dupuis J, Tardif JC, Cernacek P, et al. 1999. Cholesterol reduction rapidly improves endothelial function after acute coronary syndromes. The RECIFE (reduction of cholesterol in ischemia and function of the endothelium) trial. Circulation, 99:3227-33.

Engler MB, Engler MM, Ursell PC, et al. 1994. Vasorelaxant properties of n-3 polyunsaturated fatty acids in aortas from spontaneously hypertensive and normotensive rats. J Cardiovasc Risk, 1:75-80.

Engler MM, Engler MB, Malloy M, et al. 2004. Docosahexaenoic acid restores endothelial function in children with hyperlipidemia: results from the EARLY study. Int J Clin Pharmacol Ther, 42:672-9.

Faulx MD, Wright AT, Hoit BD, et al. 2003. Detection of endothelial dysfunction with brachial artery ultrasound scanning. Am Heart $J$, 145:943-51.

Flachs P, Mohamed-Ali V, Horakova O, et al. 2006 Polyunsaturated fatty acids of marine origin induce adiponectin in mice fed a high-fat diet. Diabetologia, 2006;49:394-7.

Fulton D, Gratton JP, McCabe TJ, et al. 1999. Regulation of endotheliumderived nitric oxide production by the protein kinase Akt. Nature, 399:597-601.

Galley HF and Webster NR.2004. Physiology of the endothelium. Br J Anaesth, 93:105-13.

[GISSI] Gruppo Italiano per lo Studio della Sopravvivenza nell'Infarto miocardico. 1999. Dietary supplementation with n-3 polyunsaturated fatty acids and vitamin $\mathrm{E}$ after myocardial infarction: results of the GISSI-Prevenzione trial. Lancet, 354:447-55
Goode GK, Garcia S, Heagerty AM, et al. 1997. Dietary supplementation with marine fish oil improves in vitro small artery endothelial function in hypercholesterolemic patients: a double-blind placebo-controlled study. Circulation, 96:2802-7.

Gupta M, Singh N, Verma S, et al. 2006. South Asians and cardiovascular risk: what clinicians should know. Circulation, 113:e924-9.

Hernandez-Perera O, Pérez-Sala D, Navarro-Antolín J, et al. 1998. Effects of the 3-hydroxy-3-methylglutaryl-CoA reductase inhibitors, atorvastatin and simvastatin, on the expression of endothelin-1 and endothelial nitric oxide synthase in vascular endothelial cells. J Clin Invest, 101:2711-19.

Laufs U, La Fata V, Plutzky J, et al. 1998. Upregulation of endothelial nitric oxide synthase by HMG CoA reductase inhibitors. Circulation, 97:1129-35.

Leung WH, Lau CP, Wong CK, et al. 1993 Beneficial effect of cholesterollowering therapy on coronary endothelium-dependent relaxation in hypercholesterolaemic patients. Lancet, 341:1496-500.

Liao JK and Laufs U. 2005. Pleiotropic effects of statins. Ann Rev Pharmacol Toxicol, 45:89-118.

McVeigh GE, Brennan GM, Johnston GD, et al. 1993. Dietary fish oil augments nitric oxide production or release in patients with type 2 (noninsulin-dependent) diabetes mellitus. Diabetologia, 36:33-8.

Mori TA, Watts GF, Burke V, et al. 2000. Differential effects of eicosapentaenoic acid and docosahexaenoic acid on vascular reactivity of the forearm microcirculation in hyperlipidemic, overweight men. Circulation, 102:1264-9.

[NCEP] Executive Summary of the Third Report of The National Cholesterol Education Program (NCEP) Expert Panel on Detection, Evaluation, And Treatment of High Blood Cholesterol In Adults (Adult Treatment Panel III). 2001. JAMA, 285:2486-97.

Newman WP, Middaugh JP, Propst MT, et al. 1993. Atherosclerosis in Alaska Natives and non-natives. Lancet, 341:1056-7.

Omori H, Nagashima H, Tsurumi Y, et al. 2002. Direct in vivo evidence of a vascular statin: a single dose of cerivastatin rapidly increases vascular endothelial responsiveness in healthy normocholesterolaemic subjects. Br J Clin Pharmacol, 54:395-9.

Sheu WH, Juang BL, Chen YT, et al. 1999. Endothelial dysfunction is not reversed by simvastatin treatment in type 2 diabetic patients with hypercholesterolemia. Diabetes Care, 22:1224-5.

Sorensen KE, Celermajer DS, Spiegelhalter DJ, et al. 1995. Non-invasive measurement of human endothelium dependent arterial responses: accuracy and reproducibility. Br Heart J, 74:247-53.

Staniloae C, Mandadi V, Kurian D, et al. 2006.Pioglitazone improves endothelial function in non-diabetic patients with coronary artery disease. Cardiology, 108:164-9.

Tagawa H, Shimokawa H, Tagawa T, et al. 1999. Long-term treatment with eicosapentaenoic acid augments both nitric oxide-mediated and non-nitric oxide-mediated endothelium-dependent forearm vasodilatation in patients with coronary artery disease. J Cardiovasc Pharmacol, 33:633-40.

ter Avest E, Abbink EJ, Holewijn S, et al. 2005. Effects of rosuvastatin on endothelial function in patients with familial combined hyperlipidaemia (FCH). Curr Med Res Opin, 21:1469-76.

Treasure CB, Klein JL, Weintraub WS, et al. 1995. Beneficial effects of cholesterol-lowering therapy on the coronary endothelium in patients with coronary artery disease. $N$ Engl J Med, 332:481-7.

Van de Ree MA, Huisman MV, de Man FH, et al. 2001. Impaired endothelium-dependent vasodilation in type 2 diabetes mellitus and the lack of effect of simvastatin. Cardiovasc Res, 52:299-305.

van Etten RW, de Koning EJ, Honing ML, et al. 2002. Intensive lipid lowering by statin therapy does not improve vasoreactivity in patients with type 2 diabetes. Arterioscler Thromb Vasc Biol, 22:799-804.

Vane JR, Anggard EE, Botting RM, et al. 1990. Regulatory functions of the vascular endothelium. $N$ Engl J Med, 323:27-36.

Yusuf S, Hawken S, Ounpuu S, et al. 2004. INTERHEART Study Investigators. Effect of potentially modifiable risk factors associated with myocardial infarction in 52 countries (the INTERHEART study): case-control study. Lancet, 364:937-52. 
